\title{
Nonequilibrium Reshaping of Polymersomes via Polymer Addition
}

\author{
Yongjun Men, Wei Li, Yingfeng Tu, Fei Peng, Geert-Jan A. Janssen, Roeland J. M. Nolte, ${ }^{\circledR}$ \\ and Daniela A. Wilson*(1) \\ Institute for Molecules and Materials, Radboud University, Heyendaalseweg 135, 6525 AJ Nijmegen, The Netherlands
}

Supporting Information

\begin{abstract}
Polymersomes are a class of artificial liposomes, assembled from amphiphilic synthetic block copolymers, holding great promise toward applications in nanomedicine. The diversity in polymersome morphological shapes and, in particular, the precise control of these shapes, which is an important aspect in drug delivery studies, remains a great challenge. This is due to a lack of general methodologies that can be applied and the inability to capture the morphologies at the nanometer scale. Here, we present a methodology that can accurately control the shape of polymersomes via the addition of polyethylene glycol (PEG) under nonequilibrium conditions. Various

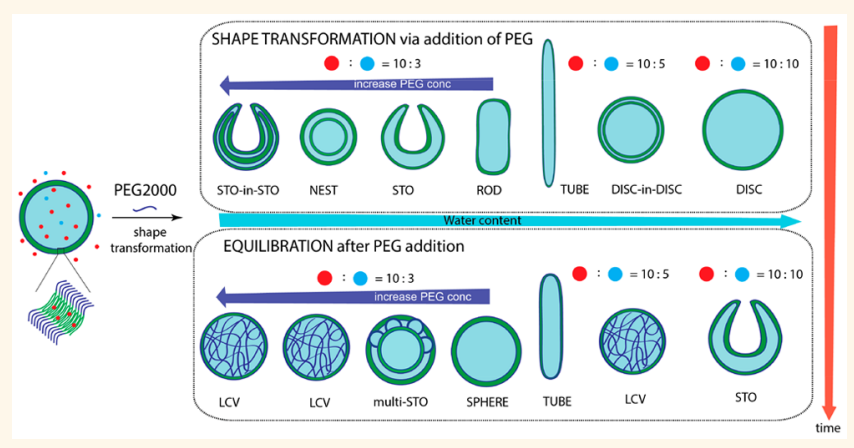
shapes including spheres, ellipsoids, tubes, discs, stomatocytes, nests, stomatocyte-in-stomatocytes, disc-in-discs, and large compound vesicles (LCVs) can be uniformly captured by adjusting the water content and the PEG concentration. Moreover, these shapes undergo nonequilibrium changes in time, which is reflected in their phase diagram changes. This research provides a universal tool to fabricate all shapes of polymersomes by controlling three variables: water content, PEG concentration, and time. The use of the biofriendly polymer PEG enables the application of this methodology in the field of nanomedicine.
\end{abstract}

KEYWORDS: nonequilibrium, polymersome, shape transformation, polymer addition, disc-in-disc, time-programed

ell shape is of key importance in cell growth and cell differentiation and, as such, has been crucial in the evolution of life. For instance, it is important in the morphogenesis of tissue during the development of the living body. Furthermore, it may help a species escape from its predators. ${ }^{1}$ The dynamic assembly of the cytoskeleton is the result of out-of-equilibrium reactions in the cell and is accompanied by shape changes, which are needed for environmental adaptation. ${ }^{1}$ Out-of-equilibrium assemblies are systems that require a continuous supply energy to persist. If the energy supply stops, the system would fall apart and end up in a thermodynamic minimum state nearby. ${ }^{2-4}$ Liposomes can spontaneously exhibit various shapes, which are reminiscent of biological cell morphologies, which have inspired scientists to fabricate vesicles (synthetic liposomes) of different shapes and compositions as cell mimetic systems in order to understand the mechanism of liposomal shape change. ${ }^{5}$ Variations in mechanical stress, temperature, $\mathrm{pH}$, or osmotic shocks and in membrane composition are capable of inducing drastic changes in liposomes, ranging from nearly spherical shapes to discocytes, stomatocytes, starfish, or pears. ${ }^{6,7}$ However, the dynamic nature of the phospholipid membrane makes these shape transitions transient due to the lack of stabilization by the cytoplasm in the cell and the fact that the cytoskeleton has glassy properties, limiting an accurate shape control.

Polymersomes assembled from amphiphilic synthetic block copolymers demonstrate enhanced membrane stiffness and provide great possibilities for tailoring both the chemical design of the membrane and its physical properties (such as stiffness, zeta-potential, and adhesiveness), which allows for good control over the shape and function of the aggregate. ${ }^{8-16}$ The shape of a polymersome is expected to affect its interaction with the cell, which is important for its flow properties, its cellular uptake, and immune regulation, as is the case for the biconcave disc-like red blood cell. ${ }^{17-21}$ Therefore, it is of great importance to be able to accurately reshape a polymersome and to understand the mechanism of the interconversions of the various shapes. Several studies have explored the possibilities to reshape polymersomes via osmotic pressure or a change in chemical structure. ${ }^{22-35}$ Block copolymers bearing liquid-crystalline side chains assembled to ellipsoids, tubes, and polyhedral shapes of polymersomes via

Received: June 17, 2019

Accepted: November 7, 2019

Published: November 7, 2019 


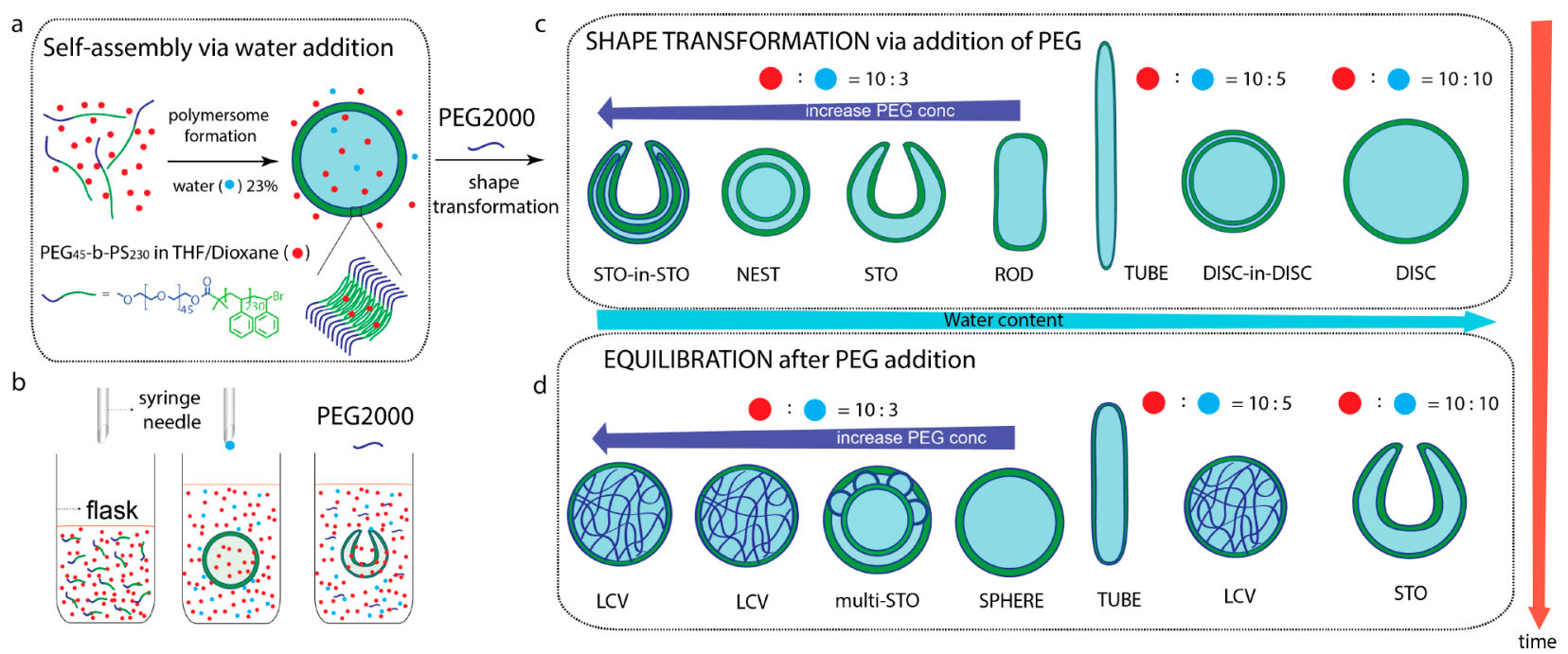

Figure 1. Overview of the nonequilibrium self-assembly approach. (a) Structure of the PEG-b-PS block copolymer and the formation of a polymersome from this compound. The red and blue dots represent organic solvent and water molecules, respectively. (b) Scheme of the self-assembly and PEG-induced shape transformation process. (c) Shapes captured after addition of PEG 2000 at the time of 1 min. Light blue arrow points to higher water content, and the dark blue arrow represents the PEG2000 concentration, which gradually changes from 0.01 to $5 \mathrm{~g} / \mathrm{L}$. (d) All of the shapes show different equilibrium behavior over time (red arrow).

solvent control. ${ }^{33,34}$ Spherical polymersomes, assembled from poly(ethylene glycol)-block-polystyrene (PEG-b-PS) block copolymers, deflate into flat discs and bowl-shaped stomatocytes via dialysis of the polymersome suspensions in mixtures of organic solvents against pure water, leading to a decrease of the inner volume of the aggregates. ${ }^{29}$ Chemical cross-linking of the polymersomes induced a shape transformation from spheres to rods. ${ }^{36}$ Our group has previously reported on the shaping of polymersomes into predictable morphologies via nonequilibrium self-assembly with the objective to mechanistically understand and predict polymersome shape changes to low-energy states. Unfortunately, only a few morphologies, such as discs, rods, and stomatocytes, were observed. ${ }^{37}$ Hence, a complete overview of the shape transformations of polymersomes and their transition from low to high energy states in order to capture unique morphologies in the nanometer range that have not been observed before is still lacking.

Herein, we report a new nonequilibrium methodology to transform spherical PEG- $b$-PS polymersomes into a variety of shapes, such as rods, tubes, discs, stomatocytes, nests, disc-indiscs, stomatocytes-in-stomatocytes (sto-in-sto), and large compound vesicles, which can be kinetically trapped at any stage of their transformation. Our former approach for obtaining a controlled shape transformation was only based on osmotic pressure changes to push further the polymersome transformation beyond the low-energy stomatocyte state to generate other shapes. ${ }^{37}$ In the present methodology, we combined additives, time, and nonequilibrium conditions as parameters for shape control. The polymersomes are initially formed by adding water to a block copolymer solution in a mixture of tetrahydrofuran (THF) and dioxane. Our approach for obtaining a controlled shape transformation is to ensure that the polymersomes only experience one single equilibrium state at the moment they are formed. Extra addition of water pushes the polymersome out of osmotic equilibrium by storing energy in the membrane. The membrane flexibility and permeability can be tuned by the water fraction in the polymersome solution, which is possible because of the amorphous glassy nature of the PS part. The PS membrane used in our studies is composed of 230 repeating units, which limits its mobility, resulting in much longer transition time scales (i.e., on the order of hours) than in the case of liposomes (seconds). This prolonged time scale gives the possibility to kinetically trap every intermediate morphology by freezing the PS membrane via quickly adding excess water. The use of PEG as a fusogen has been recently demonstrated by our group, and its ability to induce a shape change in the polymersome can already be realized with a trace amount $(0.005 \mathrm{wt} \%)$ of this compound, compared to $>15$ wt $\%$ for the liposome system, which is possible because the low permeability of the membrane amplifies the osmotic pressure. ${ }^{31}$ In this paper, we show that the bending energy of the polymersome can be regulated by tuning the rigidity of the PS membrane, allowing good control of the nonequilibrium shape transformation and the possibility to capture various morphologies over time, leading to a complete phase diagram of the shape transformations.

\section{RESULTS AND DISCUSSION}

Self-Assembly via Water Addition. Polymersomes were made by first dissolving $10 \mathrm{mg}$ of $\mathrm{PEG}_{45}-b-\mathrm{PS}_{230}$ in $1 \mathrm{~mL}$ of $\mathrm{THF} /$ dioxane $=4: 1(\mathrm{v} / \mathrm{v})$, followed by slowly adding water at a rate of $1 \mathrm{~mL} / \mathrm{h}$ (Figure $1 \mathrm{a}, \mathrm{b})$. The assembled polymersomes were formed when the ratio of water and organic solvent approached 1:4 (v/v). At this point, the solvent compositions inside and outside the polymersomes are equal (i.e., the inner and outer osmotic pressures are equal), setting this as the equilibrium point. Continuous addition of water into the solution will push the self-assembled system to nonequilibrium as an osmotic pressure builds up over the membrane. The extra water addition makes the PS membrane more rigid and less permeable. As previously observed, ${ }^{21}$ when the water content is $50 \mathrm{vol} \%$, most of the spherical polymersomes change into rods after 4 days because the organic solvent flows out faster than water flows in, resulting in a volume decrease. The system changes back to a spherical morphology after 8 days (inflow of water releases the bending energy), indicating that the 

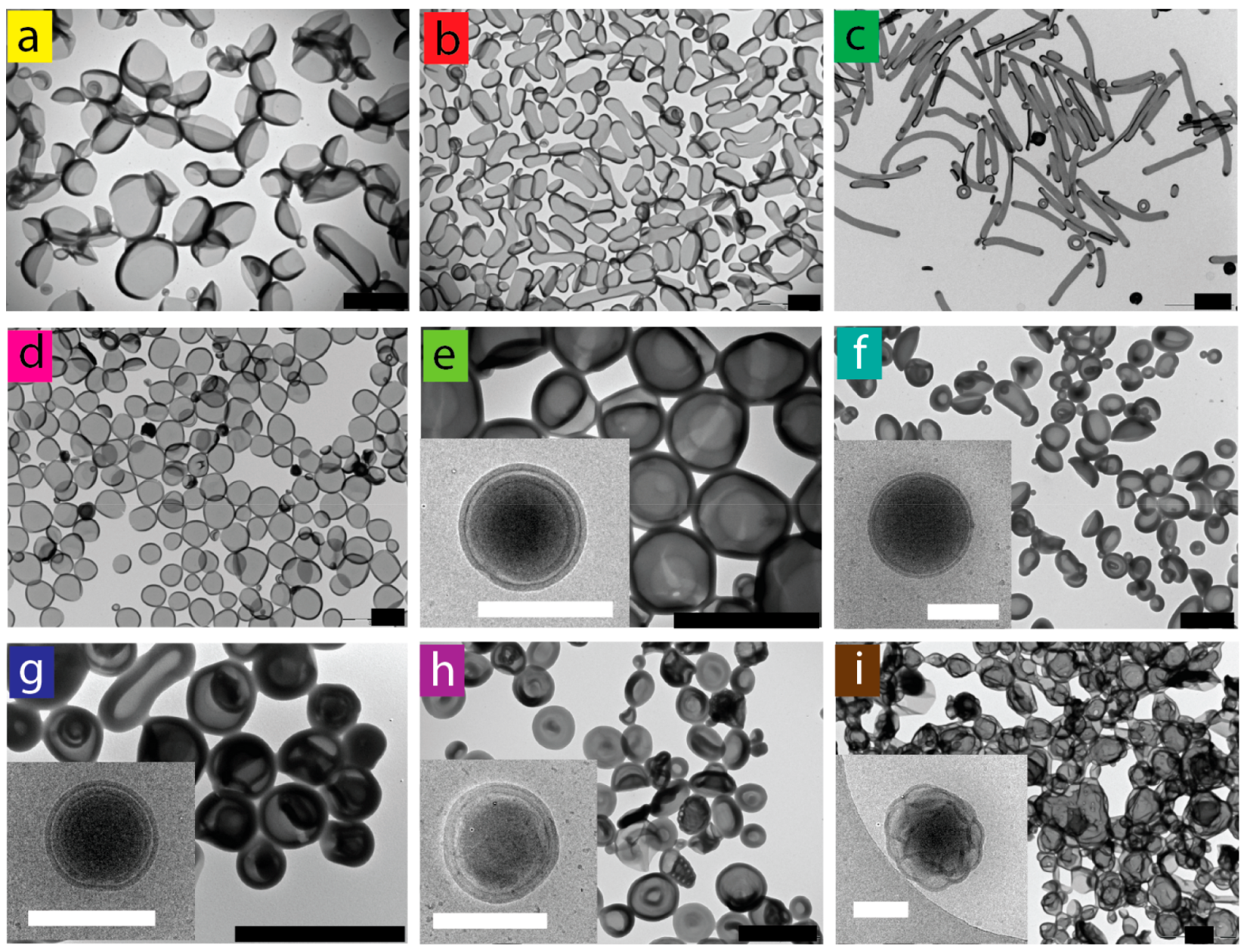

Figure 2. TEM and cryo-TEM (insets) images of polymersome morphologies recorded after 1 min, as obtained after the addition of different concentrations of PEG2000. (a) Spherical vesicles (PEG2000 concentration, water content: $0.005 \mathrm{~g} / \mathrm{L}, 23 \mathrm{vol} \%$ ), (b) ellipsoids (0.02 g/L, 23 vol \%), (c) tubes (0.05 g/L, 33 vol \%), (d) discs (0.1 g/L, 50 vol \%), (e) stomatocytes ( $0.5 \mathrm{~g} / \mathrm{L}, 33$ vol \%), (f) nests (1 g/L, $23 \mathrm{vol} \%)$, (g) sto-in-stos ( $5 \mathrm{~g} / \mathrm{L}, 23 \mathrm{vol} \%)$, (h) disc-in-discs $(5 \mathrm{~g} / \mathrm{L}, 33 \mathrm{vol} \%)$, (i) large compound vesicles ( $25 \mathrm{~g} / \mathrm{L}, 23$ vol \%). The colors of the symbols match the ones in the phase diagram in Figure 3. Scale bar: black, $1 \mu \mathrm{m}$; white, $500 \mathrm{~nm}$.

nonequilibrium shape change is driven by osmotic pressure. However, the increase of water addition leads to an increase of the membrane rigidity and a decrease of the permeability, which limits the range over which the osmotic pressure can be built up. This small range indicates that only very limited morphologies (rods, discs, and stomatocytes) are accessible with this method. Furthermore, the flexibility and mobility of the polymer chains are considerably reduced, preventing spontaneous curvature from changing and membrane fusion from taking place.

Shape Transformation via PEG Addition. To explore new paths of polymersome shape change, PEG was selected for enhancing the osmotic pressure and the interaction with the polymersome membrane, as it has been demonstrated before to act as a fusogen of liposomes due to its osmophobic association effect. ${ }^{38}$ This effect demonstrates that the PEG molecules are excluded from the region adjacent to the lipid vesicle surface (exclusion layer), which induces osmotic stress onto the vesicles, resulting in membrane fusion. Based on our previous report, ${ }^{29}$ PEG with a molecular weight of 2000 was chosen as the fusogen as relatively large molecules can be excluded more effectively from the exclusion layer adjacent to the membrane surface than small molecules, which is seen as the "mechanical stress model". ${ }^{39}$ As shown in Figure 1b, when the volume of water reached a certain value $(0.3-2 \mathrm{~mL}), 200$ $\mu \mathrm{L}$ of the polymersome suspension was transferred to a centrifuge tube, followed by the addition of $10 \mu \mathrm{L}$ of an aqueous PEG2000 solution to the suspension. The shape of polymersomes immediately changed to a variety of morphologies depending on the PEG2000 concentration and water content (Figure 1c). We expect that the added PEG2000 polymer molecules in the solution will mostly stay outside of the polymersome membranes due to their inability to penetrate the thick hydrophobic PS membranes. This PEG addition procedure pushes the polymersomes even further away from the equilibrium situation when compared to the experiments in which only water is added. The morphology was fixed by quenching $200 \mu \mathrm{L}$ of polymersome solution with $1 \mathrm{~mL}$ of water for transmission electron microscopy (TEM) measurement. All shapes that were captured are presented in Figure 2, together with the preparation conditions, such as water content and PEG2000 concentration that were used for the shape transformation. This information on the morphologies was used to construct a phase diagram, which is shown in Figure 3. The solid line in this figure means that only one type of shape was observed in the particular region, and the dashed line is a mixture of both shapes. 


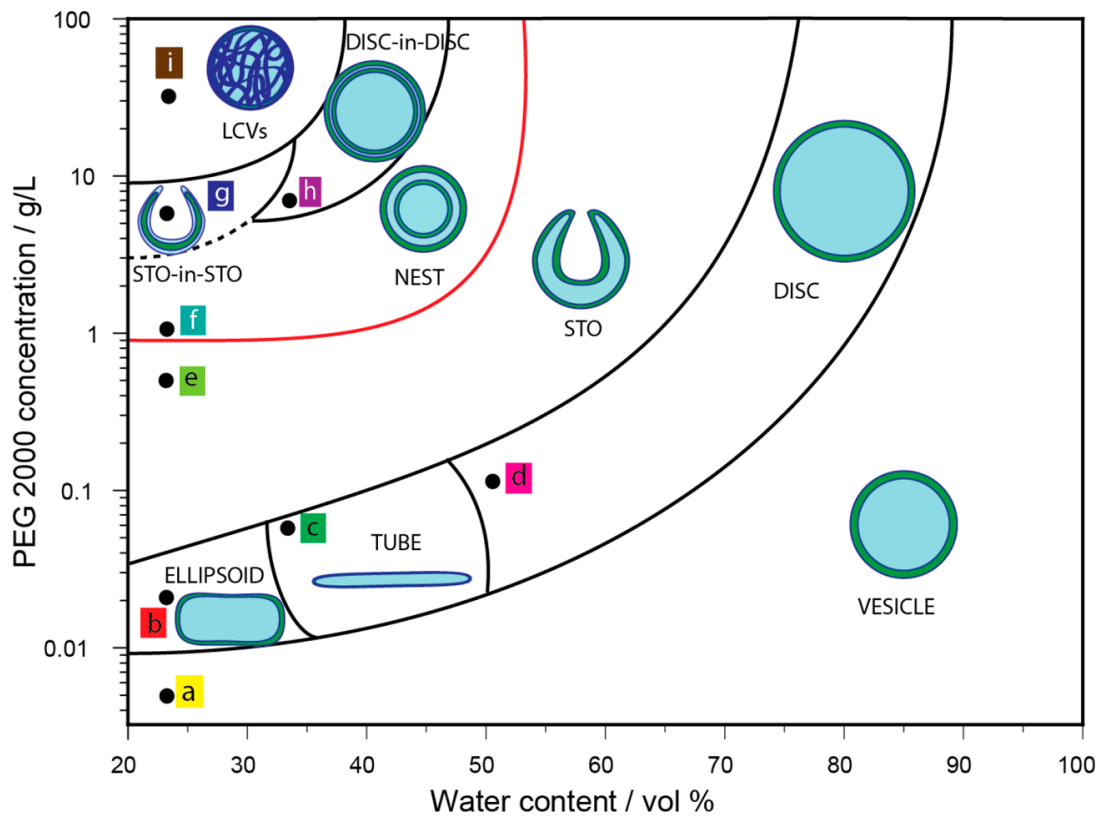

Figure 3. Phase diagram of polymersome morphologies before equilibrium. The points in the picture correspond to the images in Figure 2. The solid line means that only one shape is observed in the region; the dashed line means a mixture of two shapes in that area. The red line is defined as being the threshold concentration line for fusion.

The observed morphology changes can be categorized into two classes: (i) shape transitions with membrane fusion and (ii) transitions without membrane fusion, mainly depending on the PEG2000 concentration. When the PEG2000 concentration was above a threshold value (see the upward pointing line of the nested vesicles in the phase diagram; Figure 3, red line), the membranes started to fuse, forming nested vesicles, stomatocyte-in-stomatocytes (sto-in-stos), discocyte-in-discocytes (disc-in-discs), and large compound vesicles (LCVs) (Figure 2f-i). Below the PEG2000 threshold value, the membranes remained stable, forming ellipsoids, tubes, discs, and stomatocytes (Figure $2 \mathrm{~b}-\mathrm{e}$ ). The different shape changes can be explained in terms of osmotic pressure and membrane permeability.

Spherical shapes (Figure 2a) are stable and sustained in the region of the phase diagram, where the PEG2000 concentration remains below $0.01 \mathrm{~g} / \mathrm{L}$ and the water content is low (the situation of a highly permeable membrane) because such PEG concentrations generate very small osmotic pressures, which can easily equilibrate due to the high permeability of the membrane. Spherical shapes are also found at high PEG2000 concentrations and high water contents (the situation of a low permeable membrane) when the rigid membrane prevents water and organic solvent to leak out. A slight increase of the PEG2000 concentration at low water content leads to an increase in the induced osmotic pressure, resulting in a shape transformation from sphere to ellipsoid. The ellipsoid shape will gradually elongate to a tube by continuous increasing the osmotic pressure and water content. In the region of high organic solvent content (leading to a membrane with high permeability and low rigidity), the osmotic pressure is high enough to increase the curvature, meanwhile pushing much more organic solvent out, resulting in a shape transformation to stomatocytes. In the region of low organic solvent, the shape changes to a disc as it requires a higher bending energy for the transformation to a stomatocyte, whereas only a small volume of organic solvent is available to leak out. As can be seen in
Figure 3, stomatocytes are obtained over a large region and are well under control as they are situated at the lowest energy state. Moreover, we found that the size of the opening of stomatocytes can be manipulated by adjusting the water content. As shown in Figure S1, the average diameters of the open mouth of the stomatocytes decreased from 414 to 177 $\mathrm{nm}$ and further to $21 \mathrm{~nm}$ when the water content fell from 60 to $50 \mathrm{vol} \%$ and then to $33.3 \mathrm{vol} \%$.

Nested shape structures were formed when, with the assistance of the PEG2000, a larger reduction of the inner volume was possible, coupled with a fusion of the mouth. During this fusion process, a part of the PEG2000 solution would be entrapped inside the nested shape, leading to an increase in the organic solvent content. Higher osmotic pressure will push the organic solvent out for the second time, forming a disc-in-disc shaped structure. As demonstrated in the phase diagram, this disc-in-disc shape possesses a high bending energy, hence, capture of such morphologies is only possible when the membrane is relatively rigid. When the membrane is too flexible (water content $23 \mathrm{vol} \%$ ), these disc and disc-in-disc shapes are not obtainable; instead, stomatocytes and sto-in-sto shapes are formed directly. If the concentration of PEG2000 is greater than $10 \mathrm{~g} / \mathrm{L}$, the polymersome corona cannot be stabilized by electrostatic and steric effects, leading to membrane fusion and to large compound vesicles. Shorter PS chain $\left(\mathrm{PEG}_{45}-\mathrm{PS}_{190}\right)$ polymersome transformed to similar shapes as $\mathrm{PEG}_{45}-\mathrm{PS}_{230}$ at the same concentration (Figure $\mathrm{S} 3$ ), but the $\mathrm{PEG}_{45}-b$ - $\mathrm{PS}_{160}$ polymersome membrane is too flexible to be fused to irregular shapes even at low PEG2000 concentrations (Figure S2).

Equilibration after PEG Addition. The process after PEG addition continuously induces a shape change until equilibrium is reached (Figure 1d). This shape change mainly involves three processes. First, after the first balancing of the osmotic pressure, the polymersome will reinflate by the simultaneous inflow of water and organic solvent to decrease the bending energy, while maintaining the osmotic balance. Second, PEG 

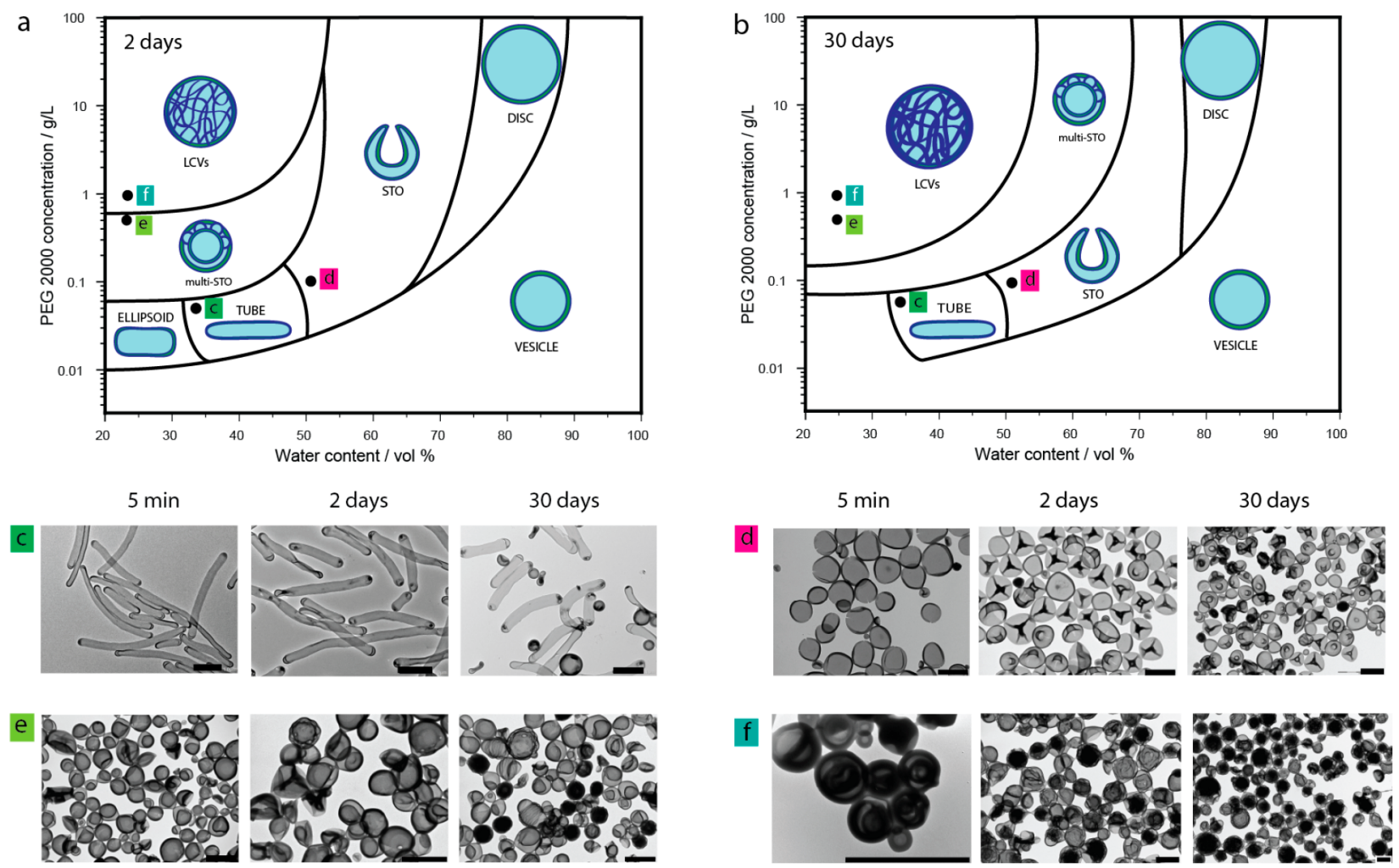

Figure 4. Morphology changes of polymersomes after equilibration. The phase diagrams of polymersome morphologies after equilibration for 2 days (a) and 30 days (b). At selected conditions, which are the same as in Figure 2, the long tubes (c), discs (d), stomatocytes (e), and nests (f) change to short/wide tubes, multiopened stomatocytes, and LCVs, after equilibration times of 2 days and 30 days. The colors match the labels in Figures 2 and 3. Scale bar: $1 \mu \mathrm{m}$.

molecules diffusing in and out of the polymersome will continuously assist in the fusing/aggregation of the polymersomes. Third, the polymer chains will rearrange in order to release the bending energy. These three factors synergistically work on the self-assembled system through a nonequilibrium process via different morphologies to adapt to the minimum energy path. Figure $4 a, b$ presents the phase diagram change after 2 days and 30 days. At low water content, the membrane is flexible and slightly permeable to water, allowing inflow of both water and organic solvent, resulting in the shape transformation from long/narrow tubes $(\sim 5 \mu \mathrm{m}$ long) to shorter/wider tubes $(\sim 2 \mu \mathrm{m}$ long). The shorter/wider tubes cannot transform back to spheres because they reached the kinetic equilibrium state (Figure 4c). As the shape change from sphere to disc is discontinuous, the excess energy generated from the osmotic pressure is stored in the PS membrane via polymer chain tension. This tension is then slowly alleviated in time by chain rearrangements, which can also be seen as bending energy. Thus, a high concentration of PEG creates a high osmotic pressure, which flattens the polymersome to a disc shape. The extra bending energy can be slowly released via a shape change to stomatocyte (Figure $4 d$ ). In the same context, but when PEG is present in high concentration and the membrane is plasticized by the large amount of organic solvent, all morphologies, such as sto-in-sto, disc-in-disc, and nest shapes, equilibrate to large composite vesicles, due to the interaction of PEG with the membrane of the polymersome (Figure 4f). Smaller PEG concentrations and a highly flexible membrane will push the stomatocytes to form multiopened stomatocytes (Figure 4e). Furthermore, once the membranes are fused together, it is impossible at this condition to separate them back to polymersomes again, which would need the system to overcome extremely high surface energies.

\section{CONCLUSION}

In summary, we have demonstrated a universal methodology for reshaping nanometer-scale polymersomes into various new morphologies in a controllable manner via PEG-guided nonequilibrium self-assembly. Furthermore, we have constructed the complete phase diagrams of all transitions. Each of the produced morphologies is highly uniform, and the structures are fully reproducible, including the stomatocyte openings, which can be strictly controlled. The latter is of great importance and can be used as a soft method for encapsulation purposes (e.g., of drug molecules). All structures started from a spherical morphology and transformed to various shapes via different routes whose mechanisms have been investigated. Low concentrations of PEG2000 below the threshold concentration (red line in Figure 3) induce only osmotic pressure over the membrane, resulting in the transition from the spherical shape to ellipsoid, tube, disc, and stomatocyte. Above the threshold concentration, PEG2000 not only creates a high osmotic pressure but also acts as a fusogen of the membrane due to the effect of osmophobic association, which assists the shape change to nest, sto-in-sto, disc-in-disc, and LCV. The rigidity of the membrane, which is tuned by the ratio of water and organic solvent regulates the path of the shape changes and the kinetics, allowing each shape during the transformation to be to captured in time spans varying from hours to days. We demonstrated that all of these shape transformation procedures are kinetic traps and nonequilibrium at short time scales. The kinetic pathway strongly 
depends on parameters such as the organic solvent (THF and dioxane) type, ratio, and water content, as well the added PEG2000. These differently shaped polymersomes are promising candidates for applications as nanocontainers or nanomachines. As we already demonstrated, the nonbiodegradable PS could be replaced by other biodegradable polymers such as poly(D,L-lactide), which exhibits a similar glassy behavior at room temperature as PS, offering opportunities for the practical application of the aggregates in drug delivery.

\section{EXPERIMENTAL METHODS}

Materials. All reagents and chemicals were purchased from SigmaAldrich and used as received. Milli-Q water (18.1 M $\Omega$ ) was used throughout the experiments. The molecular weights of the block copolymers were measured with a Shimadzu Prominence GPC system equipped with a PL gel $5 \mu \mathrm{m}$ mixed D column (Polymer Laboratories) and differential refractive index and UV $(254 \mathrm{~nm})$ detectors. THF was used as an eluent with a flow rate of $1 \mathrm{~mL} / \mathrm{min}$. NMR spectra were performed on a Varian Inova 400 spectrometer with $\mathrm{CDCl}_{3}$ as a solvent. Transmission electron microscopy samples were prepared in the following way: a solution of a sample $(6 \mu \mathrm{L})$ was air-dried on a carbon-coated Cu TEM grid (200 mesh). A TEM JEOL 1010 microscope at an acceleration voltage of $60 \mathrm{kV}$ was used to perform the measurements. A sonicator VWR USC300TH was used for the sonication experiments at room temperature. A JEOL 2100 cryo-transmission electron microscope was used for a detailed characterization. PEG-b-PS was synthesized as described in our previous paper. ${ }^{31}$

Preparation of Polymersomes. The procedure as described in our previous literature report was used. ${ }^{22}$ A typical procedure is as follows: $\mathrm{PEG}_{45}-b-\mathrm{PS}_{230}(10 \mathrm{mg})$ was dissolved in a solvent mixture of tetrahydrofuran (THF) and 1,4-dioxane (dioxane) ( $1 \mathrm{~mL}, 4: 1$ by volume) in a $15 \mathrm{~mL}$ capped vial with a magnetic stirrer. After the compounds were dissolved for $1 \mathrm{~h}$ at room temperature, a syringe pump equipped with a syringe with a needle was calibrated to deliver water at a speed of $1 \mathrm{~mL} / \mathrm{h}$. The needle from the syringe was inserted into the vial of which the cap was replaced with a rubber septum. An amount of $0.3 \mathrm{~mL}$ of water was pumped into the organic solution with vigorous stirring $(900 \mathrm{rpm})$. After the water addition was finished, 50 $\mu \mathrm{L}$ of the suspension was dropped at once into $1 \mathrm{~mL}$ of pure water with stirring, which ensured a rapid quenching of the PS domain within the bilayer of the polymersomes.

PEG Addition Methodology. A polymersome solution $(200 \mu \mathrm{L}$, $0.8 \mathrm{~g} / \mathrm{L}$ ) in a mixture of THF/dioxane water $=10: 3$ (or 10:5, 10:10, $10: 20,10: 30$ and 10:40) by volume was loaded in a $1.5 \mathrm{~mL}$ Eppendorf centrifugation tube. An amount of $10 \mu \mathrm{L}$ (or another amount) of a PEG2000 aqueous solution $(1,10,100 \mathrm{~g} / \mathrm{L})$ was added to the polymersome suspension $(200 \mu \mathrm{L})$ with a frequency of 1200 . After 1 min, $20 \mu \mathrm{L}$ of the polymersome suspension was taken out to be quenched by $1 \mathrm{~mL}$ of ultrapure water.

Equilibrium. The polymersome suspension was left in the shaker while the temperature was kept constant $\left(20^{\circ} \mathrm{C}\right)$. At the desired time, $20 \mu \mathrm{L}$ of the polymersome suspension was quenched into $1 \mathrm{~mL}$ of water located in a $1.5 \mathrm{~mL}$ centrifuge tube. The tube was centrifuged at $10000 \mathrm{rpm}$ for $5 \mathrm{~min}$ to remove the added PEG. The polymersomes aggregated at the bottom of the tube were redispersed by addition of $1 \mathrm{~mL}$ of water, then one drop of the suspension was taken to prepare the TEM sample.

\section{ASSOCIATED CONTENT}

\section{(5 Supporting Information}

The Supporting Information is available free of charge on the ACS Publications website at DOI: 10.1021/acsnano.9b04740.

Materials and instrumentation; synthesis methods of PEG- $b$-PS and polymersomes, as well shape transformation via PEG addition methodology; TEM images of stomatocytes with various mouth openings; shape change of polymersomes assembled from different PS lengths (PDF)

\section{AUTHOR INFORMATION}

\section{Corresponding Author}

*Tel.: +31 (0)24 36 52185. E-mail: d.wilson@science.ru.nl. ORCID $\odot$

Roeland J. M. Nolte: 0000-0002-5612-7815

Daniela A. Wilson: 0000-0002-8796-2274

\section{Author Contributions}

Y.M. and W.L. contributed equally. Y.M. and D.A.W. designed the experiments and wrote the manuscript. Y.M., W.L., Y.T., and F.P. performed the experiments and analyzed the results. G.-J.A.J. performed the cryo-transmission electron microscope measurements. Y.M., W.L., Y.T., F.P., R.J.M.N., and D.A.W. interpreted the results.

\section{Notes}

The authors declare no competing financial interest.

\section{ACKNOWLEDGMENTS}

This research was supported by the European Research Council via an ERC Starting grant (307679 StomaMotors) and NWO Chemische Wetenschappen (VIDI Grant 723.015.001) to D.A.W. Further support was received from the Ministry of Education, Culture and Science (Gravitation program 024.001.035). W.L. acknowledges funding from the China Scholarship Council. Y.M. acknowledges fruitful discussions with Prof. Jan van Hest.

\section{REFERENCES}

(1) Pollard, T. D.; Cooper, J. A. Actin, a Central Player in Cell Shape and Movement. Science 2009, 326, 1208-1212.

(2) Mattia, E.; Otto, S. Supramolecular Systems Chemistry. Nat. Nanotechnol. 2015, 10, 111-119.

(3) Boekhoven, J.; Brizard, A. M.; Kowlgi, K. N. K.; Koper, G. J. M.; Eelkema, R.; van Esch, J. H. Dissipative Self-Assembly of a Molecular Gelator by Using a Chemical Fuel. Angew. Chem., Int. Ed. 2010, 49, $4825-4828$.

(4) van Esch, J. H.; Klajn, R.; Otto, S. Chemical Systems Out of Equilibrium. Chem. Soc. Rev. 2017, 46, 5474-5475.

(5) Rooijen, N. V.; Sanders, A. Liposome Mediated Depletion of Macrophages: Mechanism of Action, Preparation of Liposomes and Applications. J. Immunol. Methods 1994, 174, 83-93.

(6) Berndl, K.; Käs, J.; Lipowsky, R.; Sackmann, E.; Seifert, U. Shape Transformations of Giant Vesicles: Extreme Sensitivity to Bilayer Asymmetry. EPL 1990, 13, 659.

(7) Hotani, H. Transformation Pathways of Liposomes. J. Mol. Biol. 1984, 178, 113-120.

(8) Ahmed, F.; Photos, P. J.; Discher, D. E. Polymersomes as Viral Capsid Mimics. Drug Dev. Res. 2006, 67, 4-14.

(9) Thevenot, J.; de Oliveira, H.; Sandre, O.; Pourtau, L.; Andres, E.; Miraux, S.; Thiaudiere, E.; Berra, E.; Lecommandoux, S. Multifunctional Polymersomes for Cancer Theranostics. J. Controlled Release 2013, 172, e44-e45.

(10) Wang, J.; Liu, K.; Xing, R.; Yan, X. Peptide Self-Assembly: Thermodynamics and Kinetics. Chem. Soc. Rev. 2016, 45, 5589-5604.

(11) Zhang, L.; Eisenberg, A. Morphogenic Effect of Added Ions on Crew-Cut Aggregates of Polystyrene-b-Poly(acrylic acid) Block Copolymers in Solutions. Macromolecules 1996, 29, 8805-8815.

(12) Discher, B. M.; Won, Y.-Y.; Ege, D. S.; Lee, J. C.-M.; Bates, F. S.; Discher, D. E.; Hammer, D. A. Polymersomes: Tough Vesicles Made from Diblock Copolymers. Science 1999, 284, 1143-1146. 
(13) Pochan, D. J.; Chen, Z.; Cui, H.; Hales, K.; Qi, K.; Wooley, K. L. Toroidal Triblock Copolymer Assemblies. Science 2004, 306, 9497.

(14) van Hest, J. C. M.; Delnoye, D. A. P.; Baars, M. W. P. L.; van Genderen, M. H. P.; Meijer, E. W. Polystyrene-Dendrimer Amphiphilic Block Copolymers with a Generation-Dependent Aggregation. Science 1995, 268, 1592-1595.

(15) Zhang, L.; Eisenberg, A. Multiple Morphologies of "Crew-Cut" Aggregates of Polystyrene- $b$-Poly(acrylic acid) Block Copolymers. Science 1995, 268, 1728-1731.

(16) Zhang, L.; Yu, K.; Eisenberg, A. Ion-Induced Morphological Changes in "Crew-Cut" Aggregates of Amphiphilic Block Copolymers. Science 1996, 272, 1777-1779.

(17) Balmert, S. C.; Little, S. R. Biomimetic Delivery with Microand Nanoparticles. Adv. Mater. 2012, 24, 3757-3778.

(18) Kolhar, P.; Anselmo, A. C.; Gupta, V.; Pant, K.; Prabhakarpandian, B.; Ruoslahti, E.; Mitragotri, S. Using Shape Effects to Target Antibody-Coated Nanoparticles to Lung and Brain Endothelium. Proc. Natl. Acad. Sci. U. S. A. 2013, 110, 10753-10758.

(19) Petros, R. A.; DeSimone, J. M. Strategies in the Design of Nanoparticles for Therapeutic Applications. Nat. Rev. Drug Discovery 2010, 9, 615-627.

(20) Perry, J. L.; Herlihy, K. P.; Napier, M. E.; DeSimone, J. M. PRINT: A Novel Platform Toward Shape and Size Specific Nanoparticle Theranostics. Acc. Chem. Res. 2011, 44, 990-998.

(21) Rothenbuhler, J. R.; Huang, J.-R.; DiDonna, B. A.; Levine, A. J.; Mason, T. G. Mesoscale Structure of Diffusion-Limited Aggregates of Colloidal Rods and Disks. Soft Matter 2009, 5, 3639-3645.

(22) Men, Y.; Peng, F.; Tu, Y.; van Hest, J. C. M.; Wilson, D. A. Methods for Production of Uniform Small-Sized Polymersome with Rigid Membrane. Polym. Chem. 2016, 7, 3977-3982.

(23) Salva, R.; Le Meins, J.-F.; Sandre, O.; Brûlet, A.; Schmutz, M.; Guenoun, P.; Lecommandoux, S. Polymersome Shape Transformation at the Nanoscale. ACS Nano 2013, 7, 9298-9311.

(24) Meeuwissen, S. A.; Kim, K. T.; Chen, Y.; Pochan, D. J.; van Hest, J. C. M. Controlled Shape Transformation of Polymersome Stomatocytes. Angew. Chem., Int. Ed. 2011, 50, 7070-7073.

(25) Abdelmohsen, L. K. E. A.; Williams, D. S.; Pille, J.; Ozel, S. G.; Rikken, R. S. M.; Wilson, D. A.; van Hest, J. C. M. Formation of WellDefined, Functional Nanotubes via Osmotically Induced Shape Transformation of Biodegradable Polymersomes. J. Am. Chem. Soc. 2016, 138, 9353-9356.

(26) Blanazs, A.; Madsen, J.; Battaglia, G.; Ryan, A. J.; Armes, S. P. Mechanistic Insights for Block Copolymer Morphologies: How Do Worms Form Vesicles? J. Am. Chem. Soc. 2011, 133, 16581-16587.

(27) Deng, Z.; Qian, Y.; Yu, Y.; Liu, G.; Hu, J.; Zhang, G.; Liu, S. Engineering Intracellular Delivery Nanocarriers and Nanoreactors from Oxidation-Responsive Polymersomes via Synchronized Bilayer Cross-Linking and Permeabilizing Inside Live Cells. J. Am. Chem. Soc. 2016, 138, 10452-10466.

(28) Du, J.; Tang, Y.; Lewis, A. L.; Armes, S. P. pH-Sensitive Vesicles Based on a Biocompatible Zwitterionic Diblock Copolymer. J. Am. Chem. Soc. 2005, 127, 17982-17983.

(29) Kim, K. T.; Zhu, J.; Meeuwissen, S. A.; Cornelissen, J. J. L. M.; Pochan, D. J.; Nolte, R. J. M.; van Hest, J. C. M. Polymersome Stomatocytes: Controlled Shape Transformation in Polymer Vesicles. J. Am. Chem. Soc. 2010, 132, 12522-12524.

(30) Ladmiral, V.; Semsarilar, M.; Canton, I.; Armes, S. P. Polymerization-Induced Self-Assembly of Galactose-Functionalized Biocompatible Diblock Copolymers for Intracellular Delivery. J. Am. Chem. Soc. 2013, 135, 13574-13581.

(31) Men, Y.; Li, W.; Janssen, G.-J.; Rikken, R. S. M.; Wilson, D. A. Stomatocyte in Stomatocyte: A New Shape of Polymersome Induced via Chemical-Addition Methodology. Nano Lett. 2018, 18, 20812085.

(32) Zhu, J.; Zhang, S.; Zhang, K.; Wang, X.; Mays, J. W.; Wooley, K. L.; Pochan, D. J. Disk-Cylinder and Disk-Sphere Nanoparticles via A Block Copolymer Blend Solution Construction. Nat. Commun. 2013, 4, 2297.
(33) Wong, C. K.; Mason, A. F.; Stenzel, M. H.; Thordarson, P. Formation of Non-Spherical Polymersomes Driven by Hydrophobic Directional Aromatic Perylene Interactions. Nat. Commun. 2017, 8, 1240.

(34) Wong, C. K.; Martin, A. D.; Floetenmeyer, M.; Parton, R. G.; Stenzel, M. H.; Thordarson, P. Faceted Polymersomes: A Sphere-toPolyhedron Shape Transformation. Chem. Sci. 2019, 10, 2725-2731.

(35) Wong, C. K.; Stenzel, M. H.; Thordarson, P. Non-Spherical Polymersomes: Formation and Characterization. Chem. Soc. Rev. 2019, 48, 4019-4035.

(36) van Oers, M. C. M.; Rutjes, F. P. J. T.; van Hest, J. C. M. Tubular Polymersomes: A Cross-Linker-Induced Shape Transformation. J. Am. Chem. Soc. 2013, 135, 16308-16311.

(37) Rikken, R. S. M.; Engelkamp, H.; Nolte, R. J. M.; Maan, J. C.; van Hest, J. C. M.; Wilson, D. A.; Christianen, P. C. M. Shaping Polymersomes into Predictable Morphologies via Out-of-Equilibrium Self-Assembly. Nat. Commun. 2016, 7, 12606.

(38) Yamazaki, M.; Ito, T. Deformation and Instability of Membrane Structure of Phospholipid Vesicles Caused by Osmophobic Association: Mechanical Stress Model for the Mechanism of Poly(Ethylene Glycol)-Induced Membrane Fusion. Biochemistry 1990, 29, 1309-1314.

(39) Yamazaki, M.; Ohnishi, S.; Ito, T. Osmoelastic Coupling in Biological Structures: Decrease in Membrane Fluidity and Osmophobic Association of Phospholipid Vesicles in Response to Osmotic Stress. Biochemistry 1989, 28, 3710-3715. 